\title{
APPLICATION D'UNE MÉTHODE DE DÉTERMINATION EXTERNE DU SEXE CHEZ LE SAUMON ATLANTIQUE (SALMO SALARL.) DANS LES RIVIĖRES DES ASTURIES (ESPAGNE)
}

\author{
E. PRÉVOST (1), V. VAUCLIN (1), J. L. BAGLINIÈRE (1), \\ F. BRANA-VIGIL (2), A.G. NICIEZA (2)
}

(1) Laboratoire d'Ecologie Hydrobiologique, Station de Physiologie et d'Ecologie des Poissons - INRA - 65, Route de St Brieuc - 35042 RENNES CEDEX, FRANCE.

(2) Departamento de Biologia de Organismos y Sistemas, Universidad de Oviedo, c/Jesùs Arias de Velasco s/n - 33005 OVIEDO, ESPAÑA.

\section{RÉSUMÉ}

Une méthode de détermination externe du sexe basée sur le dimorphisme sexuel de la longueur relative de la mâchoire supérieure est appliquée chez le Saumon Atlantique (Salmo salar L.). 78 adultes capturés en juin et juillet 1990 dans trois rivières des Asturies (Nord de l'Espagne) sont étudiés. L'Analyse Linéaire Discriminante sur un échantillon de 63 poissons donne un pourcentage d'erreurs de moins de $10 \%$. Les résultats sont comparés à ceux obtenus sur d'autres cours d'eaux de l'aire de répartition du Saumon atlantique et sont discutés en fonction de la composition de l'échantillon.

Mots-clés : Saumon atlantique, Salmo salar, sexe, dimorphisme sexuel, détermination du sexe, Espagne.

\section{USE OF A TECHNIQUE TO DETERMINE THE SEX \\ OF ATLANTIC SALMON (SALMO SALAR L.) \\ IN THE RIVERS OF ASTURIAS (SPAIN)}

\begin{abstract}
A technique to determine the sex of Atlantic salmon (Salmo salar L.) from the sexual dimorphism of the relative length of the upper jaw bone was used. A total of 78 adults caught in june and july 1990 in three spanish rivers of Asturias (Spain) were studied. Linear Discriminant Analysis from a sample of 63 fish gave an error percentage less than $10 \%$. Results were compared to those obtained in other rivers of the distribution area of Atlantic salmon and were discussed in relation to the structure of the sample.
\end{abstract}

Key-words : Atlantic salmon, Salmo salar, sexual dimorphism, sex determination, Spain.

\section{INTRODUCTION}

Le rapport des sexes et surtout le nombre de femelles sont des paramètres indispensables à connaître dans les études de dynamique de population et/ou pour la gestion des stocks de Saumon atlantique (Salmo salar L.). La méthode de détermination du sexe la plus fiable et la plus utilisée demeure l'examen direct des gonades après autopsie (BAGLINIÉRE et al., 1987). 
Pour la Truite de mer (Salmo trutta), LEBAIL (1981) a proposé une technique basée sur le dimorphisme sexuel de la longueur de la mâchoire supérieure, qui permet de sexer des individus vivants.

Cette technique a été testée avec succès sur des Saumons atlantique adultes de la rivière North-Esk (Ecosse) (MAISSE et BAGLINIĖRE, 1986) et de certains cours d'eau québécois (MAISSE et al., 1988). Sa mise en œuvre pratique sur le terrain est aisée, et elle semble présenter un bon niveau de fiabilité (BAGLINIËRE etal., 1989), même si elle n'a été actuellement éprouvée que sur un nombre assez réduit de cours d'eau.

Ainsi, il est apparu intéressant de tester à nouveau cette méthode sur des poissons adultes des rivières des Asturies (Nord de l'Espagne). La récolte des données a été largement facilitée par l'existence, sur chacune des rivières étudiées, d'un poste de déclaration des captures où de nombreuses données sont collectées. Le sexe des saumons y est déterminé par simple observation visuelle de leur morphologie externe. Le travail présenté ici peut donc aussi être considéré comme un essai de rationalisation de cette approche empirique.

\section{MATÉRIEL ET MÉTHODES}

78 saumons ont été échantillonnés entre le 5 juin et le 4 juillet 1990 sur le Narcea, le Sella et le Cares, qui sont les trois rivières les plus productives de la Province des Asturies (plus de 1.000 captures chacune les bonnes années : NICIEZA et al. (1990)) (Fig. 1). La plupart de ces poissons $(n=75)$. ont été pris à la ligne, les autres étant des individus malades récupérés morts. La répartition par rivière de l'échantillon est donnée dans le tableau I A.

La longueur à la fourche de chaque saumon adulte a été mesurée (LF en millimètres, précision de $5 \mathrm{~mm}$ ) ainsi que celle de la longueur de la mâchoire supérieure ( $L M$ en millimètres, précision de $1 \mathrm{~mm}$ ), à l'aide d'un compas à pointe sèche (Fig. 2). Des écailles ont été prélevées et l'âge de mer a été déterminé selon les normes établies par le Conseil International pour l'Exploration de la Mer (ANONYME, 1984). La répartition de l'échantillon étudié selon l'âge de mer est donnée dans le tableau I $\mathrm{B}$.

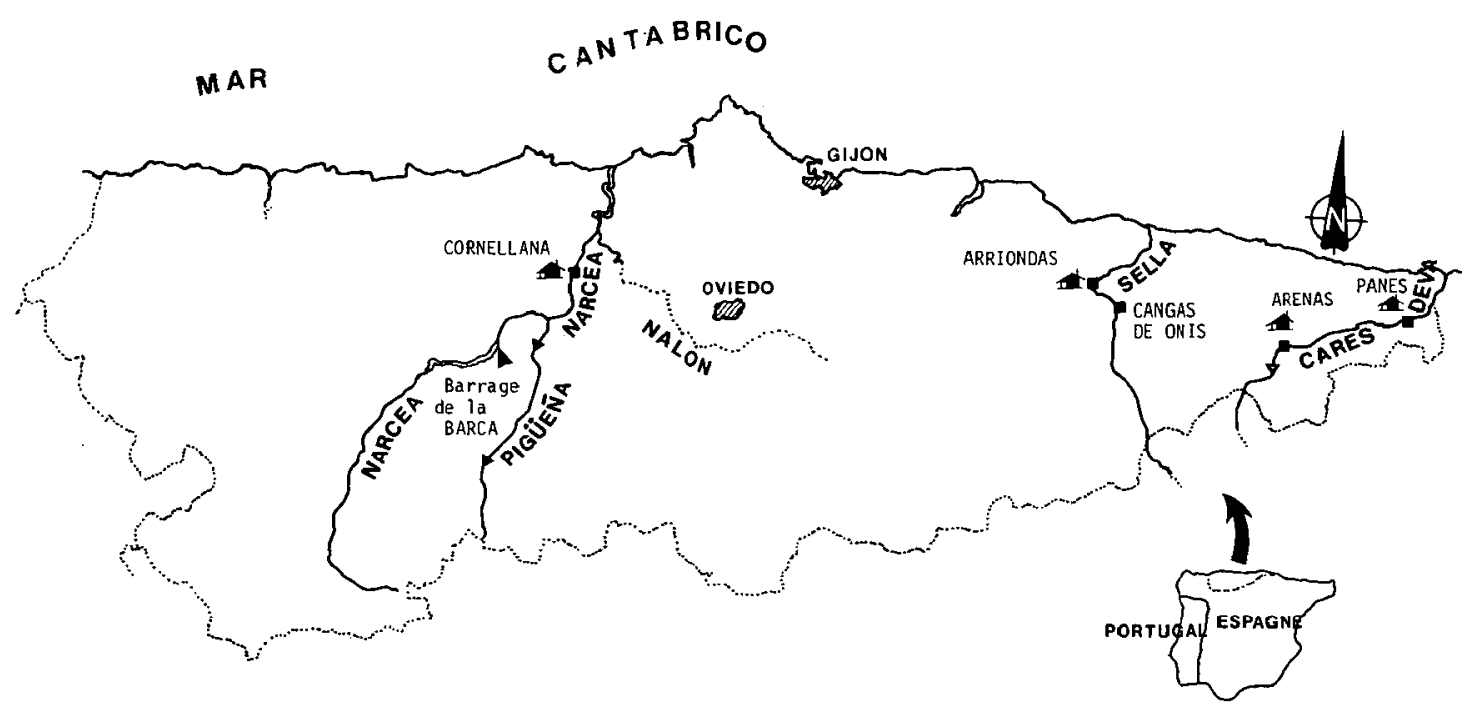

Figure 1 : Situation des rivières échantillonnées dans la Province des Asturies (Nord de l'Espagne).

1 = poste de déclaration de captures.

Figure 1 : Map of sampled rivers in the Asturias Country (Northern Spain). $I=$ site of catches recording. 
Tableau I : Répartition de l'échantillon de saumons étudiés et fiabilité de la détermination du sexe

A - par rivière

$B$ - par âge de mer

Table I : Distribution of the sample of salmon studied and reliability for sex determination A - according to river

$B$ - according to sea-age

A

\begin{tabular}{llcccc}
\hline & RIVIĖRE & NARCEA & SELLA & CARES & TOTAL \\
SEXE & & & & & \\
\hline \multirow{2}{*}{ Certain } & Mâles & 37 & 1 & 4 & 42 \\
& Femelles & 12 & 2 & 7 & 21 \\
& TOTAL & 49 & 3 & 11 & 63 \\
& Mâles & 12 & & & \\
& Femelles & 3 & & 0 & 15 \\
\hline & TOTAL & 15 & 0 & 11 & 78 \\
\hline
\end{tabular}

B

\begin{tabular}{llrrr}
\hline & AGE DE MER & $1+$ & $2-2+$ & TOTAL \\
SEXE & & & & \\
\hline \multirow{2}{*}{ Certain } & Mâles & 35 & 6 & 41 \\
& Femelles & 6 & 16 & 22 \\
& TOTAL & 41 & 22 & 63 \\
& Mâles & 10 & 2 & 12 \\
& Femelles & 1 & 2 & 3 \\
& TOTAL & 11 & 4 & 15 \\
\hline & TOTAL GÉNÉRAL & 52 & 26 & 78 \\
\hline
\end{tabular}

La détermination du sexe a été effectuée suivant deux modalités :

- examen direct des gonades après autopsie du poisson,

- palpation digitale des organes génitaux (reconnaissance tactile des grappes ovariennes) après une légère incision pratiquée au niveau de la partie inférieure du cleithrum lorsque l'ouverture abdominale du poisson ne pouvait être effectuée (Fig. 2).

Deux expérimentateurs ont utilisé cette seconde méthode, dont l'un était peu entrainé. Les déterminations de ce dernier (15 poissons) ont été considérées comme incertaines dans les analyses qui ont suivi ; les 63 saumons de sexe certain ont donc constitué l'échantillon de base (Tableau I). Les données (longueur à la fourche, longueur de la mâchoire supérieure) ont été traitées de deux manières : 
- des régressions linéaires ont été effectuées sur l'échantillon de base, ainsi que séparément par sexe et pour chaque groupe d'âge de mer. Les droites obtenues ont été comparées deux à deux par des analyses de covariance (SCHERRER, 1984);

- l'Analyse Linéaire Discriminante (ROMEDER, 1973 ; RICHARDOT, 1985) à deux groupes (mâle et femelle) a été utilisée pour l'échantillon de base, puis pour chaque groupe d'âge de mer. On a fait intervenir une "probabilité a priori" d'affectation à chaque groupe (proportion de chaque sexe) dans les calculs de Fonction Linéaire Discriminante (RICHARDOT, loc. cit.). Chaque analyse a donné lieu à une évaluation de la règle de décision par rééchantillonnage Bootstrap (RICHARDOT, loc. cit.). Le logiciel SPAD.N (Version 1.2) a été employé.

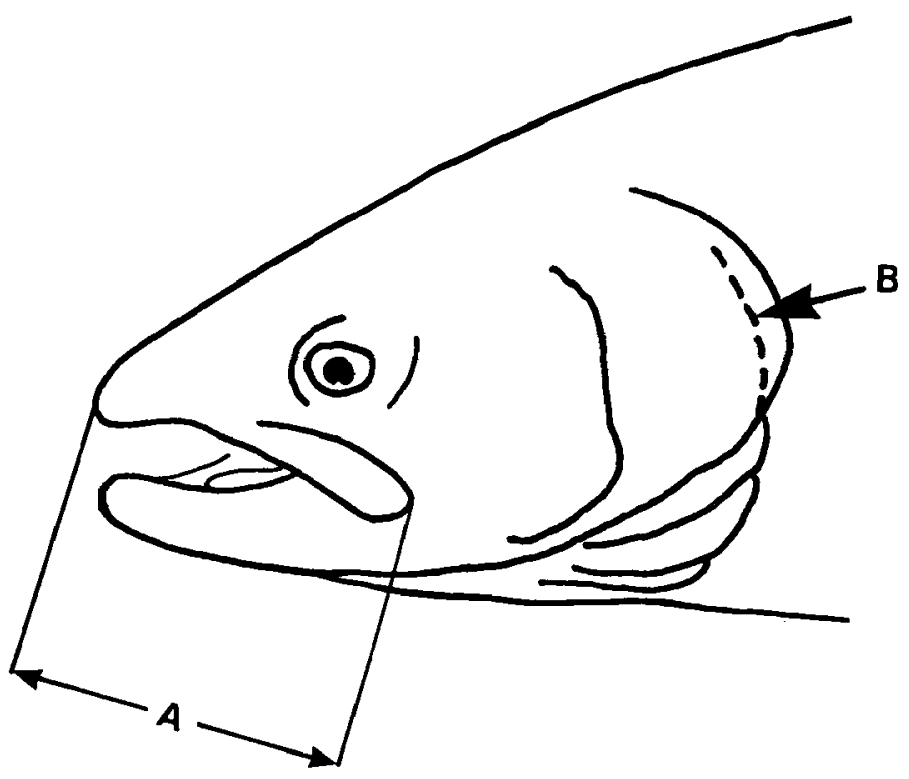

Figure 2 : Longueur de la mâchoire supérieure (A) et localisation de l'incision pratiquée pour la palpation des organes génitaux (B).

Figure 2 : Length of the upper jaw bone (A) and localization of the slight opening used for the tactile examination of reproductive organs (B).

\section{RÉSULTATS}

Sur l'échantillon de base, il existe une forte relation linéaire entre la longueur à la fourche et celle de la mâchoire $(r=0,83 ; p<0,01)$. Les régressions linéaires calculées séparément montrent qu'il est possible de distinguer les mâles des femelles à partir de la longueur relative de la mâchoire : les pentes des droites de régression de chacun des deux sexes sont significativement différentes ( $p<0,005$; Figure 3$)$.

Les droites de régression relatives aux deux groupes d'âge de mer (1 et 2 hivers marins) diffèrent significativement par leur ordonnée à l'origine $(p=0,005)$, leurs pentes ne pouvant être considérées comme distinctes au seuil de $5 \%$ (Figure 4 ).

L'Analyse Discriminante appliquée à l'échantillon de base $(n=63)$ affecte correctement $92 \%$ des poissons (Figure 5). Le rééchantillonnage Bootstrap donne des résultats pratiquement invariants quel que soit le nombre d'itérations $(40,50$, ou 70$)$; il donne une estimation du pourcentage de bien classés de $91 \%$, ce qui atteste la stabilité de la règle de décision. 
Sur les 15 poissons de sexe incertain (utilisés en tant qu'échantillon test), 5 sont mal affectés par la règle de décision établie à partir de l'échantillon de base, ce qui correspond à un taux de bien classés de $67 \%$.

La discrimination menée séparément sur les castillons et les poissons de plusieurs hivers marins donne des droites de discrimination et de séparation différentes (Figure 5), mais des pourcentages de bien classés identiques $(90 \%)$.

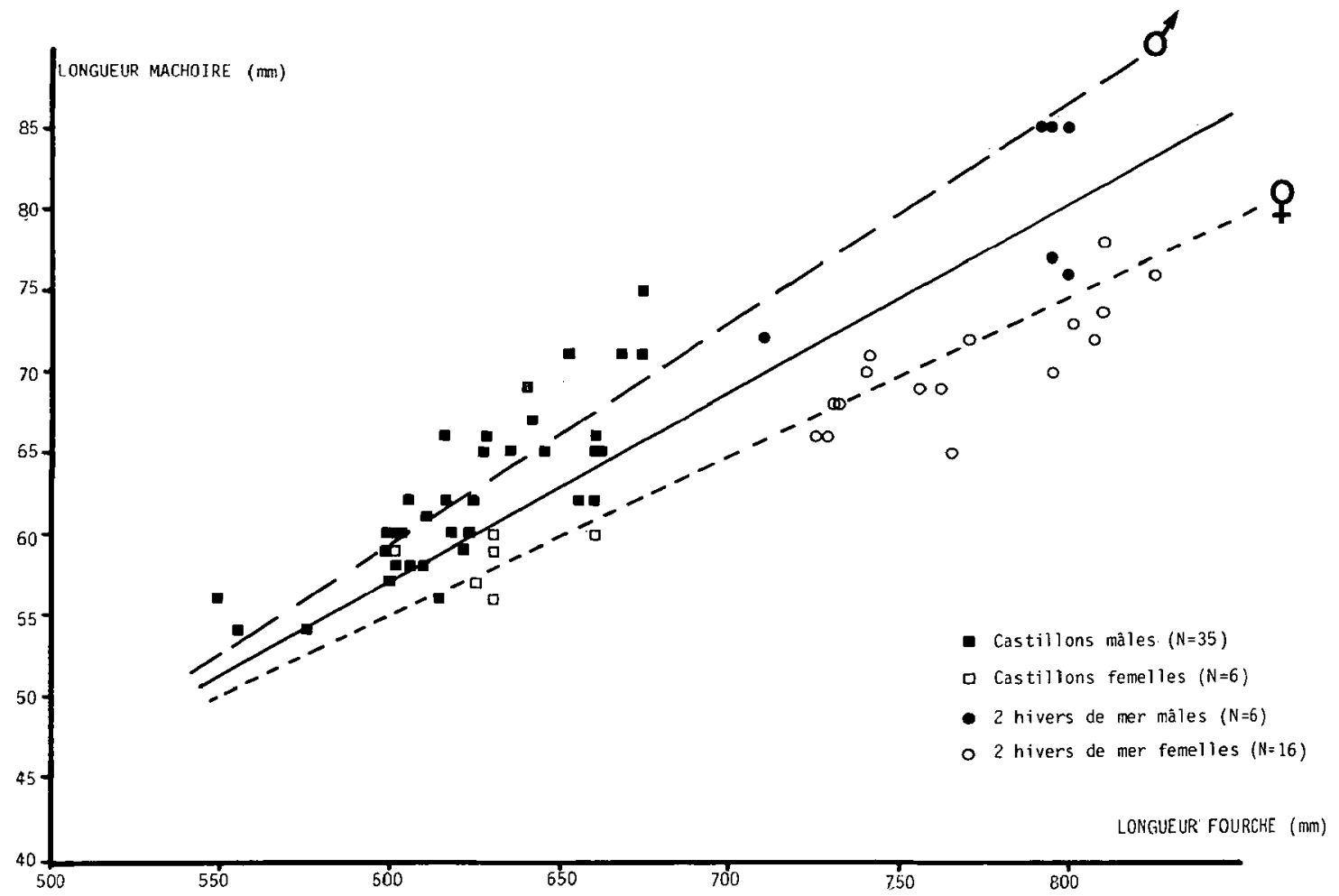

Figure 3 : Relation entre la longueur fourche (LF) et la longueur de la mâchoire supérieure (LM) pour l'échantillon total et selon le sexe. Les droites de régression figurées ont les équations suivantes :

Echantillon étudié $(n=63)$ :

$L F=8,58 L M+110,90(r=0,83)$

Mâles $(n=41)$ :

$L F=7,32 L M+169,86(r=0,92)$

Femelles $(n=22)$ :

$L F=10,63 L M+17,58(r=0,94)$

Figure 3 : Relationship between fork length (LF) ant upper jaw bone length (LM) for the studied sample and according to sex. Linear regression equations presented are the following ones :

Studied sample $(n=63)$ :

$L F=8.58 L M+110.90(r=0.83)$

Male $(n=41)$ :

$L F=7.32 L M+169.86(r=0.92)$

Female $(\mathbf{n}=\mathbf{2 2})$ :

$L F=10.63 L M+17.58(r=0.94)$ 


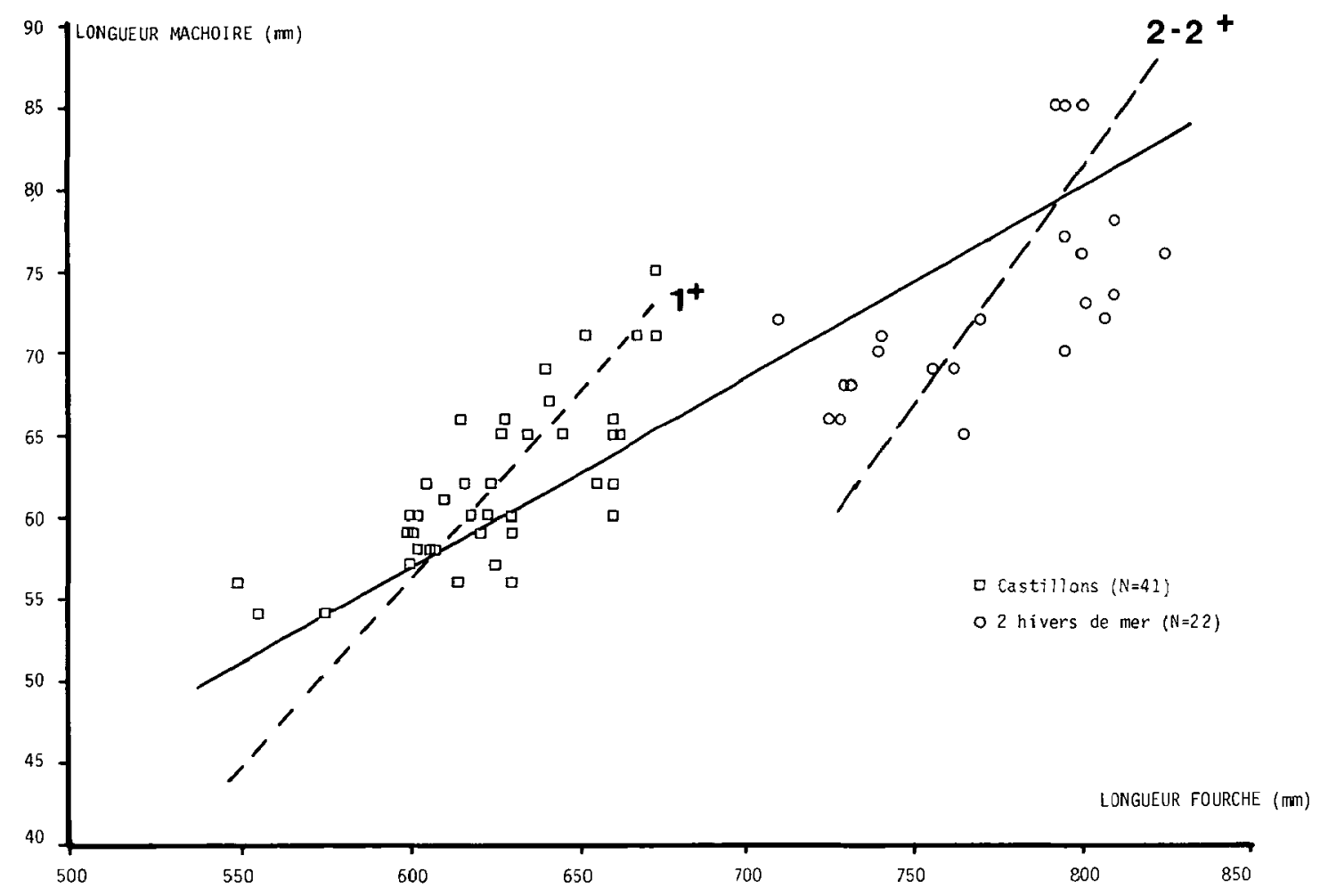

Figure 4 : Relation entre la longueur fourche(LF)et la longueur de la mâchoire supérieure pour l'échantillon total et selon l'âge de mer. Les droites de régression figurées ont les équations suivantes:

Echantillon étudié $(n=63)$ :

$L F=8,58$ LM $+110,90(r=0,83)$

Castillons $(n=41)$ :

$L F=4,34$ LM $+355,94(r=0,75)$

Rédibermarins $(n=22)$ :

$L F=3,45 \mathrm{LM}+519,97(\mathrm{r}=0 ; 67)$

Figure 4 : Relationship between fork length (LF) and upper jaw bone length (LM) for the studied sample and according to sea age. Linear regression equations presented are the following ones:

Studied sample $(n=63)$ :

$L F=8.58 L M+110.90(r=0.83)$

Grilse ( $n=41)$ :

$L F=4.34 L M+355.94(r=0.75)$

Multisea-winter fish $(n=22)$ :

$L F=3.45 L M+519.97(r=0.67)$ 


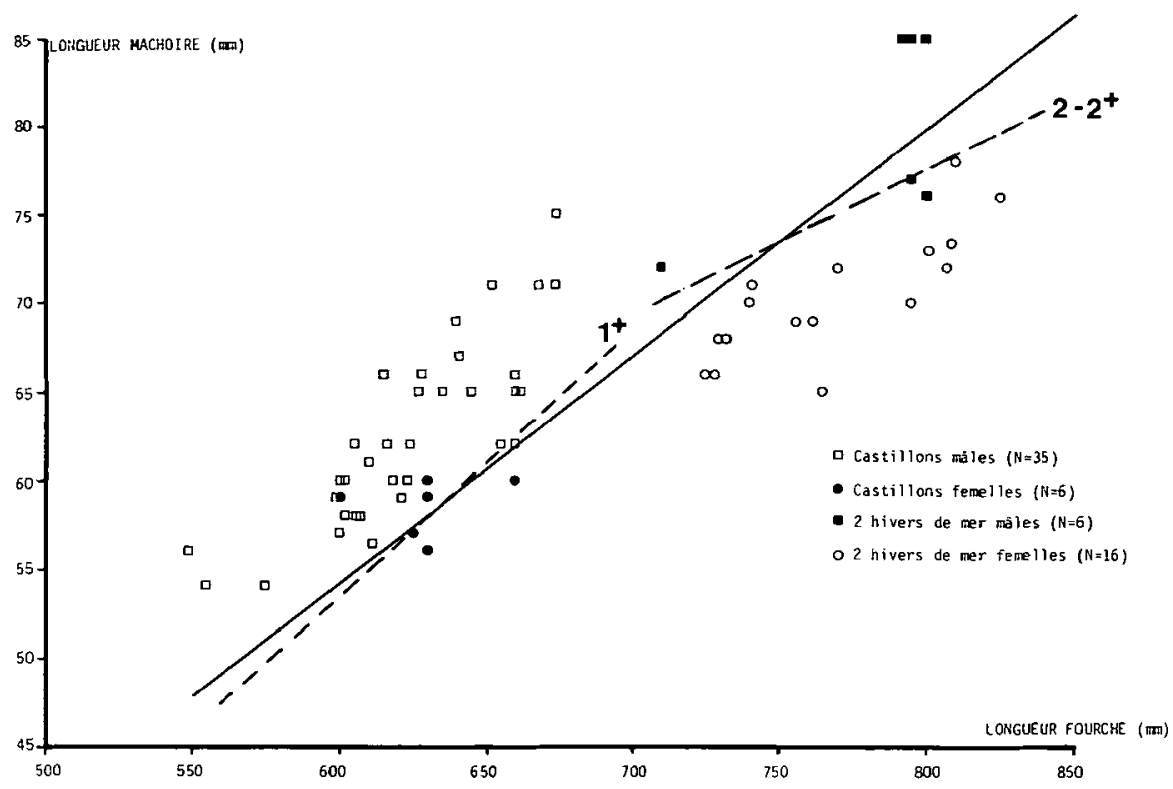

Figure 5 : Relation entre la longueur fourche(LF)et la longueur de la mâchoire supérieure (LM) et discrimination des sexes par Analyse Linéaire Discriminante pour l'échantillon étudié et selon l'âge de mer. Les équations des fonctions linéaires discriminantes (FLD) et des droites de séparation (DS) sont les suivantes :

Echantillon étudié $(n=63)$ :

$92 \%$ de bien classés

FLD Y $=-0,092 L F+0,716 L M+16,222$

DS LF $=7,757 \mathrm{LM}+175,728$

Estimation Bootstrap (70 itérations) :

$91 \%$ de bien classés.

Castillons $(n=41)$ :

$90 \%$ de bien classés

FLD $Y=-0,082 L F+0,579 L M+16,503$

DS $L F=7,036 \mathrm{LM}+200,418$

Rédibermarins $(n=22)$ :

$91 \%$ de bien classés

FLD $Y=-0,073$ LF + 0,988 LM $-17,691$

DS LF = 13,548 LM - 242,666.

Figure 5 : Relationship between fork length (LF) and upper jaw bone length (LM) and discrimination of the sex by Linear Discriminant Analysis for the studied sample and according to sea-age. The equations of linear discriminant functions (FLD) and separation lines presented (DS) are the following ones :

Studied sample $(n=63)$ :

$92 \%$ of well distributed

$F L D Y=-0.092 L F+0.716 L M+16.222$

DS LF = 7.757 LM + 175.728

Bootstrap estimation (70 iterating) :

$91 \%$ of well distributed

Grilse $(n=41)$ :

$90 \%$ of well distributed

FLD Y $=-0.082 L F+0.579 L M+16.503$

DS LF $=7.036 \mathrm{LM}+\mathbf{2 0 0 . 4 1 8}$

Multisea winterfish $(n=22)$ :

$91 \%$ of well distributed

FLD $Y=-0.073$ LF + 0.988 LM - 17.691

DS LF = 13.548 LM - 242.666. 


\section{DISCUSSION}

Comme dans le cas des poissons de la North Esk (MAISSE et BAGLINIĖRE, 1986) ou du Québec (MAISSE et al., 1988), la longueur relative de la mâchoire supérieure permet de déterminer le sexe des individus de l'échantillon de base $(n=63)$ avec un taux de succès proche de $90 \%$ (Tableau II). Par contre, les Fonctions Linéaires Discriminantes obtenues en Espagne, en Ecosse et au Québec sont différentes. L'interprétation de ces écarts est délicate. Quelques remarques peuvent cependant être faites:

Tableau II : Comparaison des résultats des études de détermination du sexe d'après la longueur de la mâchoire supérieure réalisées sur le Saumon atlantique.

Table II : Comparison of results of studies for Atlantic salmon sex determination from the relative length of upper jaw bone.

\begin{tabular}{|c|c|c|c|c|c|c|}
\hline REFERENCE & $\begin{array}{c}\text { LIEU } \\
\text { D'ETUDE } \\
\text { (Rivière, Pays) }\end{array}$ & $\begin{array}{l}\text { PERIODE } \\
\text { D'ETUDE }\end{array}$ & EFFECTIF & $\begin{array}{c}\% 2 \text { hivers } \\
\text { de mer }\end{array}$ & $\begin{array}{l}\% \text { bien } \\
\text { classés }\end{array}$ & $\begin{array}{l}\text { Equation discriminante } \\
\text { Droite de séparation }\end{array}$ \\
\hline $\begin{array}{l}\text { MAISSE et } \\
\text { BAGLINIËRE } \\
(1986)\end{array}$ & $\begin{array}{l}\text { North Esk } \\
\text { (Ecosse) }\end{array}$ & $\begin{array}{l}\text { Juillet } \\
1984\end{array}$ & 97 & $<50$ & 93 & $\begin{array}{l}Y=-0,050 L F+0,405 L M+6,618 \\
L M=0,123 L F-16,340\end{array}$ \\
\hline $\begin{array}{l}\text { MAISSE } \\
\text { et al. } \\
(1988)\end{array}$ & $\begin{array}{l}\text { Moisie, Jupiter } \\
\text { Bonaventure, } \\
\text { Grande Cascape- } \\
\text { pédia (Québec) }\end{array}$ & $\begin{array}{l}\text { Juillet } \\
1986\end{array}$ & 182 & 100 & 88 & $\begin{array}{l}Y=-0,0324 L F+0,287 L M-4,026 \\
L M=0,113 L F-14,050\end{array}$ \\
\hline $\begin{array}{l}\text { PRESENT } \\
\text { TRAVAIL }\end{array}$ & $\begin{array}{l}\text { Narcea, Sella } \\
\text { Cares } \\
\text { (Espagne) }\end{array}$ & $\begin{array}{l}\text { Juin } \\
\text { Juillet } \\
1990\end{array}$ & 63 & 35 & 92 & $\begin{aligned} Y & =-0,092 L F+0,716 L M+16,220 \\
L M & =0,134 L F-26,360\end{aligned}$ \\
\hline
\end{tabular}

- Les castillons sont majoritaires dans les échantillons espagnols et écossais et absents dans celui du Québec. La variation dans l'expression des droites de séparation et de discrimination selon l'âge de mer peut expliquer les différences observées entre les stocks européens et nord-américains.

- Les résultats obtenus peuvent être le reflet de différences morphologiques, biologiques (comme le révèle la structure superficielle des écailles (LEAR et SANDEMAN, 1980) ou génétiques (GUYOMARD, 1987) entre les stocks des deux continents. A un degré moindre les mêmes arguments peuvent être avancés en ce qui concerne les écarts observés entre les populations de la North Esk et des rivières des Asturies.

- La période d'échantillonnage est légèrement plus tardive dans le cas du Québec et de l'Ecosse. En outre la période de reproduction dans ces deux zones (BAGLINIERE et al., 1979) est plus précoce qu'en Espagne (mois de décembre-janvier). Les saumons échantillonnés au Québec et en Ecosse se trouvaient donc sans doute dans un état de maturité sexuelle plus avancée que ceux analysés dans le présent travail. Les modifications des caractéristiques morphologiques des mâles étant sous contrôle androgénique (LILEY et STACEY, 1983), ceci pourrait expliquer en partie les résultats différents obtenus pour les saumons des Asturies.

- Compte tenu de la taille des échantillons utilisés, les différences observées dans les résultats pourraient n'être que des "artefacts" d'échantillonnage.

Le pourcentage de bien classés de 15 individus de sexe incertain (utilisé comme échantillon test) est de $67 \%$. Ce résultat est très proche de celui que l'on obtient en réalisant une affectation au hasard tenant compte du rapport des sexes observé sur l'échantillon de base $(60 \%$ de bien classés, soit 1 individu mal classé de plus). Ces deux pourcentages sont très similaires, mais aussi nettement inférieurs au taux de succès estimé sur l'échantillon de base (>90\%). Ceci confirme le caractère douteux de la détermination du sexe des 15 poissons mis à l'écart, plutôt que cela ne remette en cause la fiabilité de la règle de décision mise au point sur l'échantillon de base. 
La prise en cómpte des caractéristiques de l'échantillon de base conduit cependant à nuancer les résultats présentés :

- Sa composition est déséquilibrée en terme de rapport des sexes (65\% de mâles) et de classes d'âge de mer (65\% de castillons). En outre, on observe de fortes proportions de mâles parmi les castillons autopsiés ( 35 sur 41 , soit $85 \%$ ) et de femelles parmi les rédibermarins (poissons de plusieurs hivers de mer) (15 sur 22 , soit $68 \%$ ). On peut comparer les résultats de la règle de décision $\mathrm{R} 1$ : "les castillons sont des mâles et les saumons rédibermárins des femelles" à ceux de l'Analyse Discriminante (Tableau III). Le gain global de discrimination apporté par la fonction de Fisher est faible $(+12 \%)$. Il est encore plus réduit pour les castillons (+ $8 \%$ de bien classés) alors qu'il est plus important pour les saumons de plusieurs hivers de mer ( $+23 \%$ de bien classés par rapport à R1), ces derniers étant les plus intéressants en terme de potentiel reproducteur (majorité de femelles dont la fécondité absolue est plus élevée que celle des castillons femelles). L'appréciation de ce gain ne repose cependant que sur un faible effectif $(n=22$ ).

Tableau III : Pourcentage (nombre) d'individus bien classés résultant de la règle de décision de l'Analyse Linéaire Discriminante (ALD) et de la règle R1 (les castillons sont des mâles et les saumons rédibermarins des femelles) en fonction du nombre d'hivers de mer. L'effectif total est de 63 saumons.

Table III : Percentage (number) of well determined individuals from the decision rule of Linear Discriminant Analysis (ALD) et from the rule R1 (grisle are males and multisea-winter fish females) in relation to sea age. Total sample $=63$ salmon.

\begin{tabular}{cccc}
\hline $\begin{array}{c}\text { Age de } \\
\text { mer }\end{array}$ & 1 hiver & 2 hivers & TOTAL \\
\hline ALD & $93 \%$ & $91 \%$ & $92 \%$ \\
& $(n=38)$ & $(n=20)$ & $(n=58)$ \\
R1 & $85 \%$ & $68 \%$ & $79 \%$ \\
& $(n=35)$ & $(n=15)$ & $(n=50)$ \\
\hline
\end{tabular}

- La période d'étude (début juin à début juillet), qui est celle de remontée massive des castillons, est à l'origine de la composition déséquilibrée de l'échantillon de base. Elle a également pu influer sur les résultats, puisque le dimorphisme sexuel devient d'autant plus marqué que l'on s'approche de la fraie. On peut donc se demander si la détermination du sexe serait possible en début de saison de pêche (mars et avril) avec des taux d'erreurs acceptables.

- La faible taille de notre échantillon nous a amenés à considérer conjointement des saumons de trois rivières différentes, dont une majorité provenaient du Narcea, cette rivière ayant été la plus "productive" en saumons l'année de l'étude. On peut néanmoins s'interroger sur la pertinence de ce choix, qu'il conviendrait de tester en comparant des échantillons plus conséquents pour chaque rivière. Le même problème a pu se poser dans l'étude réalisée au Québec puisqu'elle concernait quatre rivières (MAISSE et al., 1988).

Les régressions Longueur fourche-Longueur mâchoire sont significativement différentes entre les saumons d'un et de plusieurs hivers marins. L'application de l'analyse discriminante séparément à chacune des classes d'âge de mer donne des pourcentages de bien classés identiques pour les deux groupes $(90 \%)$. Ce résultat est très légèrement moins performant que celui obtenu sur l'échantillon de base. Les deux équations discriminantes donnent au total 6 individus mal classés, parmi lesquels se trouvent les 5 mal classés par la discrimination globale. II ne semble donc pas intéressant d'appliquer des discriminations séparées par âge marin sur notre échantillon, contrairement à ce que suggérait l'analyse de covariance des droites de régression séparées, même si le faible effectif de l'échantillon de base ne permet 
pas de statuer définitivement sur l'intérêt (ou non) d'une stratégie de discrimination séparée par groupe d'âge de mer.

Chez les truites de mer de la Touques (Basse-Normandie), la détermination du sexe d'après la longueur relative de la mâchoire supérieure ne donne de bons résultats qu'au delà d'une taille minimale de 40-44 cm (RICHARD, 1986). Chez le saumon coho (Oncorhynchus kisutch), BEACHAM et MURRAY (1986) ont montré que ce critère ne permet une discrimination correcte des sexes que pour des poissons de plus de $43 \mathrm{~cm}$, le dimorphisme sexuel étant plus marqué chez les grands individus. Dans notre étude, les trois castillons et les deux rédibermarins mal classés par l'Analyse Discriminante sont de taille moyenne et grande par rapport à leurs classes d'âge de mer respectives (Figure 5). Nous n'avons donc pas décelé de limite inférieure de validité de la méthode pour le saumon atlantique, confirmant les résultats des deux études précédentes sur cette espèce (MAISSE et BAGLINIĖRE, 1986 ; MAISSE et al., 1988).

Enfin, on peut se demander s'il ne serait pas possible d'obtenir une discrimination plus performante par l'utilisation simultanée de deux critères morphologiques. BEACHAM et MURRAY (1986) sur le Saumon du Pacifique, MAISSE et BAGLINIERE (1986) et NAESJE et al. (1988) sur le Saumon Atlantique ont observé un dimorphisme sexuel relatif, respectivement, à la longueur ou à la surface de la nageoire adipeuse. MAISSE et BAGLINIËRE (1986) ont montré que la discrimination des sexes était plus fiable à partir de la longueur relative de la mâchoire supérieure. Mais aucun des auteurs cités n'a testé l'utilisation simultanée de ces deux paramètres. Son intérêt pratique dépend du rapport coûtrésultat. Le coût équivaut ici à un possible alourdissement de la récolte des données, le résultat étant le gain éventuel de fiabilité apporté. L'inconvénient de la longueur de la nageoire adipeuse comme critère complémentaire est d'être difficile à mesurer de façon standardisée ; il est en effet délicat de situer sa limite antérieure.

\section{REMERCIEMENTS}

La collecte des données a été rendue possible grâce à la collaboration des pêcheurs sportifs et du personnel employé dans les postes de déclaration de captures sur le Narcea, le Sella et le Cares.

Ce travail a été financé par la Communauté Economique Européenne (Bourse COMETT), par le Conseil Régional de Bretagne et par la fondation FICYT, Principado de Asturias.

\section{BIBLIOGRAPHIE}

ANONYME, 1984. Atlantic salmon scale reading. Report of the Atlantic Salmon scale reading workshop. ICES, $17 \mathrm{p}$.

BAGLINIĖRE J.L., CHAMPIGNEULLE A., NIHOUARN A., 1979. La fraie du Saumon atlantique (Salmo salar L.) et de la Truite commune (Salmo trutta L.) sur le bassin du Scorff. Cybium 3e série, 7, 75-79.

BAGLINIĖRE J.L., PROUZET P., NIHOUARN A., PORCHER J.P., RICHARD A., 1989. Exemples de résultats de la recherche obtenus sur les populations sauvages de salmonides de Bretagne et de Basse-Normandie et applicables à leur gestion. Rev. Sci. Eau, 2, 859-874.

BAGLINIĖRE J.L., PROUZET P., PORCHER J.P., NIHOUARN A., MAISSE G., 1987. Caractéristiques générales des populations de saumon atlantique (Salmo salarL.) des rivières du Massif armoricain. In "Restauration des rivières à saumons", M. Thibault et R. Billard, Ed. INRA, Paris, 23-37.

BEACHAM T.D., MURRAY C.B., 1986. Sexual dimorphism in length of upper jaw and adipose fin of immature and maturing Pacific salmon (Oncorhynchus). Aquaculture, $58,269-276$. 
GUYOMARD R., 1987. Différenciation génétique des populations de saumon atlantique : revue et interprétation des données électrophorétiques et quantitatives. In "Restauration des rivières à saumons", M. Thibault et R. Billard, Ed. INRA, Paris, 298-308.

LEAR W.H., SANDEMAN E.J., 1980. Use of scale characters and discriminant functions for identifying continental origin of Atlantic salmon. In ICES/ICNAF Joint Investigation on North Atlantic Salmon. Rapp. P.V. Reun. Const. Int. Explor. Mer, 176, 68-75.

LEBAIL P.Y., 1981. Identification du sexe en fonction de l'état de maturité chez les poissons. Thèse Docteur Ingénieur, ENSAR, $71 \mathrm{p}$.

LILEY N.R., STACEY N.E., 1983. Hormones, pheromones and reproductive behavior in fish. In "Fish physiology", Vol. IX partie B, W.S. Hoar, D.J. Randall et E.M. Donaldson Ed., Academic Press, New York, 1-63.

MAISSE G., BAGLINIĖRE J.L., 1986. Le sexage morphologique du saumon atlantique (Salmo salar). Bull. Fr. Pêche Piscic., 300, 13-18.

MAISSE G., BAGLINIĖRE J.L., LANDRY G., CARON F., ROULEAU A., 1988. Identification externe du sexe chez le saumon atlantique (Salmo salar L.). Can. J. Zool., 66, 23122315.

NAESJE T.F., HANSEN L.P., JÄRVI T., 1988. Sexual dimorphism in the adipose fine of Atlantic Salmon, Salmo salar L. J. Fish. Biol., 33, 955-956.

NICIEZA A.G., TOLEDO M.M., BRANA F., 1990. Capturas de Salmon Atlantico (Salmo salar L.) en los rios asturianos. Variaciones de abundancia y estructura de edades de mar. Biobas. Rev. Biol. Univ. Oviedo, vol. IV, 91 p.

RICHARD A., 1986. Recherches sur la truite de mer, Salmo trutta L., en Basse-Normandie. Thèse 3e cycle, Fac. Sci., Univ. Rennes, $54 \mathrm{p}$.

RICHARDOT P., 1985. Différents outils pour la discrimination linéaire entre deux groupes : sélection des variables, bootstrap, discrimination sur coordonnées factorielles. Méthodes et logiciels. Thèse $3 e$ cycle Math. autom., Université de Paris-Dauphine, $102 \mathrm{p}$.

ROMEDER J.M., 1973. Méthodes et programmes d'analyse discriminante. Dunod, Paris, 1 vol., $274 \mathrm{p}$.

SCHERRER B., 1984. Biostatistique. Gaëtan Morin, Québec, 1 vol., 850 p. 